\title{
Aerococcus Urinae Infective Endocarditis-related Stroke: A Case Report
}

\section{Jennifer A Creed*, Divyang Patel and Larry B Goldstein}

Duke University, DUMC 2905, Durham, USA

\begin{abstract}
Aerococcus urinae is a Gram-positive, catalase-negative coccus that was first defined in 1992. A. urinae is responsible for up to $0.8 \%$ of all cases of urinary tract infection. Typical predisposing factors include male sex, age $>65$ years and pre-existing urinary pathology. A. urinae is a rare cause of endocarditis but has a high associated morbidity and mortality. Sepsis or septic shock is the primary clinical presentation but there have been several reports of cerebral ischemic events in the setting of $A$. urinae endocarditis. These case descriptions are very limited and do not include brain imaging. We describe the clinical course of a 75 year-old man who, in the setting of $A$. urinae mitral valve endocarditis, presented with symptoms and imaging findings consistent with embolic cerebral infarction. Importantly, mitral valve vegetation was detected only after transesophageal echocardiography. Given the risk of morbidity without treatment, these data support pursuing a transesophageal approach even when transthoracic echo is negative.
\end{abstract}

Keywords: Cerebrovascular disease; Stroke; Embolism; Infarction; MRI; Bacterial infections

\section{Introduction}

Aerococcus urinae is a Gram-positive, catalase-negative coccus. Occurring rarely, the few reports of cerebral ischemic events in the setting of A. urinae-mediated infective endocarditis (IE) are limited. We provide details of the case of a patient with stroke due to presumed cardiogenic embolism that was subsequently found to be caused by $A$. urinae-mediated IE.

\section{Case Report}

The patient was a 75 year old African-American man with a history of hypertension, hyperlipidemia, diabetes and chronic kidney disease who presented with a low grade fever and leukocytosis. His blood pressure was $167 / 76 \mathrm{mmHg}$, heart rate $102 \mathrm{BPM}$, and temperature $100.4^{\circ} \mathrm{F}$. A II/VI diastolic murmur was present. He was somnolent but easily arousable on presentation to the hospital. Neurological examination was otherwise notable for global aphasia, left gaze preference, right nasolabial fold flattening, and right lower extremity drift. Laboratory testing included a normal basic metabolic panel except for an elevated creatinine $(2.3 \mathrm{mg} / \mathrm{dL})$, BUN $(24 \mathrm{mg} / \mathrm{dL})$ and glucose $(277 \mathrm{mg} / \mathrm{dL})$. There was a neutrophil-predominant leukocytosis (WBC $16.5 \times 10^{9} / \mathrm{L}$ ) and normocytic anemia (Hg $\left.13.4 \mathrm{~g} / \mathrm{dL}, \mathrm{MCV} 91 \mathrm{fL}\right)$ with a normal platelet count. Urinalysis, urine culture and toxicology screening were unremarkable. Magnetic resonance imaging of the brain (Figure 1A-F) showed multiple areas of restricted diffusion in the left hemisphere within multiple vascular territories. Magnetic resonance angiography of the head and neck did not demonstrate any extraor intracranial steno-occlusive disease (images not shown). Further evaluation included a transthoracic echocardiogram which showed trivial valvular regurgitation and no evidence of an intra-cardiac shunt (images not shown). Consistent with the patient's II/VI diastolic murmur, subsequent transesophageal ultrasonography demonstrated a $1.0 \times 0.4 \mathrm{~cm}$ mobile echodensity on the anterior mitral valve leaflet (Figure 1G). There were no clinical stigmata of endocarditis.

Although our patient had several cerebrovascular risk factors, the distribution of acute ischemic injury on MRI brain was compatible with a thromboembolic source associated with native mitral valve IE. He was started on vancomycin which was continued for four days until cultures finalized without evidence of staphylococcus. Additionally, cefepime was initiated but then changed to ceftriaxone after antibiotic sensitivities became available [5]. Blood cultures from the time of initial presentation as well as two days after presentation were positive for A. urinae based on biochemical methods of detection. Blood cultures drawn on hospital day 4 resulted negative, likely in the setting of intravenous antibiotics. Urine cultures were consistently negative for A. urinae. His initial leukocytosis normalized by the time of hospital discharge. Neurologically, the patient had a persistent global aphasia with an anomia and an impaired ability to repeat, but was able to follow simple commands and answer questions with "yes" and "sometimes." He was discharged to a skilled nursing facility where he completed a 6-week course of intravenous antibiotics. At a two month outpatient follow-up visit, his neurological deficits persisted.

\section{Discussion}

Stroke related to A. urinae-mediated IE is rare. Zbinden et al described a patient who presented with mental confusion, a "left hemi-syndrome," fever, a grade IV systolic murmur and mitral valve vegetations. CT brain showed "cerebral infarction" without further description of the pathology or supporting images [1]. A second account described a left posterior cerebral artery distribution ischemic stroke in a patient with a large aortic valve vegetation; cultures of the resected valve grew A. urinae despite seronegative blood cultures [2]. Another report is of a patient presenting with a new diastolic murmur, evidence of septic emboli, and aortic valve vegetations which revealed A. urinae by DNA amplification; the patient developed hallucinations prompting a MRI brain showing "multiple ischemic lesions [3]." A report of 16 patients with $A$. urinae bacteremia included three with IE with one having a subsequent septic brain embolus [4]. Most recently

*Corresponding author: Jennifer A Creed, Duke University, DUMC 2905, Durham USA, Tel 919-681-1700; Fax 919-668-1294; E-mail: jennifer.creed@duke.edu

Received December 15, 2015; Accepted January 11, 2016; Published January 17,2016

Citation: Creed JA, Patel D, Goldstein LB (2016) Aerococcus Urinae Infective Endocarditis-related Stroke: A Case Report. J Cardiovasc Dis Diagn 4: 232. doi:10.4172/2329-9517.1000232

Copyright: ( 2016 Creed JA, et al. This is an open-access article distributed under the terms of the Creative Commons Attribution License, which permits unrestricted use, distribution, and reproduction in any medium, provided the original author and source are credited. 

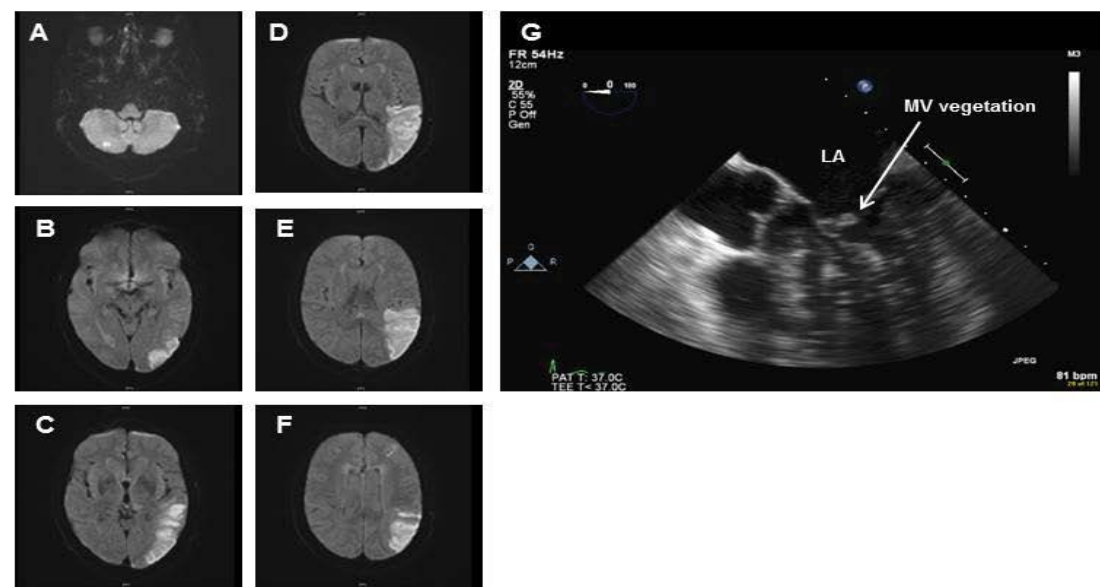

Figure 1: Magnetic resonance imaging (MRI) of the brain. Note: multiple areas of restricted diffusion consistent with acute cerebral infarctions. This includes the posterior left MCA territory involving the left parietal lobe, posterior left temporal lobe, and left lateral occipital lobe and the anterior aspect of the left middle frontal gyrus with mild associated T2 hyper-intense signal and tiny foci of restricted diffusion within the cerebellar hemispheres bilaterally. Also noted is a small left cerebellar chronic infarction and moderate T2 prolongation in the periventricular white matter and the centrum semiovale, bilaterally, most consistent with chronic microvascular ischemic disease $(\mathrm{A}-\mathrm{F})$. Transesophageal echocardiographic (TEE) image demonstrates native mitral valve (MV) endocarditis as evidenced by a $1.0 \mathrm{~cm} \times 0.4 \mathrm{~cm}$ mobile echodensity seen on the anterior mitral valve leaflet $(G)$. No thrombus is detected in the left atrium (LA).

Sunnerhagen et al. describe the association between A. urinae mediated IE and "an occlusion of the medial cerebral artery" [6].

Although the incidence of IE ranges between 1.5 and 11.6 cases per 100,000 people, the risk of IE-mediated stroke is only $0.158 \pm 0.091$ per 100,000 [7] and extremely rare when the causative organism is A. urinae. Suspicion of IE-related stroke should nonetheless remain high when the radiographic pattern of ischemic injury suggests a cardioembolic source and the patient has a fever, heart murmur, evidence of valvular disease on echocardiography or positive blood cultures. Importantly, although TTE in our patient did not show valvular disease, the TEE demonstrated a mitral valve vegetation providing a source of embolism to explain the distribution of abnormalities found on MR imaging.

It is suggested that a normal TTE effectively excludes infective endocarditis and that TEE is therefore unnecessary [8]. However, the rate of detection of valvular vegetations with TTE is less than 50-percent compared to TEE, suggesting that a transthoracic approach has limited detection capacity [9]. Given the risk of morbidity without treatment, these data support pursuing a transesophageal approach even when TTE is negative.

\section{References}

1. Zbinden R, Santanam P, Hunziker L, Leuzinger B, von Graevenitz A (1999) Endocarditis due to Aerococcus urinae: diagnostic tests, fatty acid composition and killing kinetics. Infection 27: 122-124.
2. Alozie A, Yerebakan C, Westphal B, Steinhoff G, Podbielski A (2012) Culturenegative infective endocarditis of the aortic valve due to Aerococcus urinae: a rare aetiology. Heart Lung Circ 21: 231-233.

3. Ebnöther C, Altwegg M, Gottschalk J, Seebach JD, Kronenberg A (2002) Aerococcus urinae endocarditis: case report and review of the literature. Infection 30: 310-313.

4. Senneby E, Petersson AC, Rasmussen M (2012) Clinical and microbiological features of bacteraemia with Aerococcus urinae. Clin Microbiol Infect 18: 546550 .

5. Humphries RM, Hindler JA (2014) In vitro antimicrobial susceptibility of Aerococcus urinae. J Clin Microbiol 52: 2177-2180.

6. Sunnerhagen T, Nilson B, Olaison L, Rasmussen M (2015) Clinical and microbiological features of infective endocarditis caused by aerococci. Infection.

7. Bin Abdulhak AA, Baddour LM, Erwin PJ, Hoen B, Chu VH, et al. (2014) Global and Regional Burden of Infective Endocarditis, 1990-2010: A Systematic Review of the Literature. Glob Heart 9: 131-143.

8. Barton TL, Mottram PM, Stuart RL, Cameron JD, Moir S (2014) Transthoracic echocardiography is still useful in the initial evaluation of patients with suspected infective endocarditis: evaluation of a large cohort at a tertiary referral center. Mayo Clin Proc 89: 799-805.

9. Kini V, Logani S, Ky B, Chirinos JA, Ferrari VA, et al. (2010) Transthoracic and transesophageal echocardiography for the indication of suspected infective endocarditis: vegetations, blood cultures and imaging. J Am Soc Echocardiogr 23: $396-402$ 